9 Hacke W, Albers G, Al-Rawi Y, Bogousslavsky J, Davalos A, Eliasziw M, Fischer M, Furlan A, Kaste M, Lees KR, Soehngen M, Warach S; DIAS Study Group: The Desmoteplase in Acute Ischemic Stroke Trial (DIAS): a phase II MRI-based 9-hour window acute stroke thrombolysis trial with intravenous desmoteplase. Stroke 2005;36:66-73.

10 Abciximab in acute ischemic stroke: a randomized, double-blind, placebocontrolled, dose escalation study. The Abciximab in Ischemic Stroke Investigators. Stroke 2000;31:601-609.

11 Lapchak PA, Araujo DM: Therapeutic potential of platelet glyocoprotein $\mathrm{IIb} / \mathrm{III}$ receptor antagonists in the management of ischemic stroke. Am J Cardiovasc Drugs 2003;3:87-94.

12 Selim M, Kumar S, Fink J, Schlaug G, Caplan LR, Linfante I: Seizure at stroke onset: should it be an absolute contraindication to thrombolysis? Cerebrovasc Dis 2002; 14:54-57.

13 Yasaka M, Yamaguchi T, Yonehara T, Moriyasu H: Recurrent embolization during intravenous administration of tissue plasminogen activator in acute ischemic stroke. A case report. Angiology 1994;45:481-484.

14 Bautista RED: Embolic stroke following thrombolytic therapy for myocardial infarction in a patient with preexisting ventricular thrombi. Stroke 1995;26:324-325.

15 Derex L, Nighoghossian N, Perinetti M, Honnorat J, Trouillas P: Thrombolytic therapy in acute ischemic stroke patients with cardiac thrombus. Neurology 2001;57:2122-2125.

Dr. V. Puetz, Department of Neurology

Technical University Dresden, Fetscherstrasse 74

DE-01307 Dresden (Germany)

Tel. +49 3514583565 , Fax +493514584365

E-Mailvolker.puetz@neuro.med.tu-dresden.de

Cerebrovasc Dis 2006;21:421-422

DOI: $10.1159 / 000092132$

\section{Limb-Shaking Transient Ischemic Attack Induced by Middle Cerebral Artery Stenosis}

\author{
Wei-Jian Jiang ${ }^{\mathrm{a}}$, Feng Gao ${ }^{\mathrm{a}}$, Bin Du ${ }^{\mathrm{a}}$, Trilochan Srivastava ${ }^{\mathrm{b}}$, \\ Yong-Jun Wang

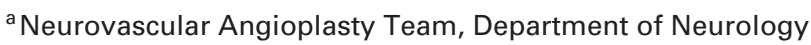 \\ and Neuroradiology, Beijing Tiantan Hospital, Capital \\ University of Medical Sciences, Beijing, China, and ${ }^{\mathrm{b}}$ All India \\ Institute of Medical Sciences, New Delhi, India
}

A 71-year-old female presented with a 3-month history of episodic shaking movements of the left leg. She had a history of hypertension for the past 10 years. These recurrent episodes would usually begin with a sensation of 'weakness' involving predominantly her left leg, and at times, the left arm. This was followed by the involuntary, focal, arrhythmic shaking movements of her left leg for about 30-45 s without spread to other limbs or body parts. The whole event would last for less than $5 \mathrm{~min}$. Her consciousness was not impaired during the attacks. These attacks would occur during prolonged standing and walking. There were $4-5$ attacks per day with increased frequency in the last month. The general physical and neurological examinations were normal. Her blood pressure was $155 / 70 \mathrm{~mm} \mathrm{Hg}$ and there was no orthostatic hypotension. The ambulatory electroencephalographic monitoring was normal. Diffusion-weighted MRI revealed hyperintense lesions in the right border zone between the anterior cerebral artery (ACA) and middle
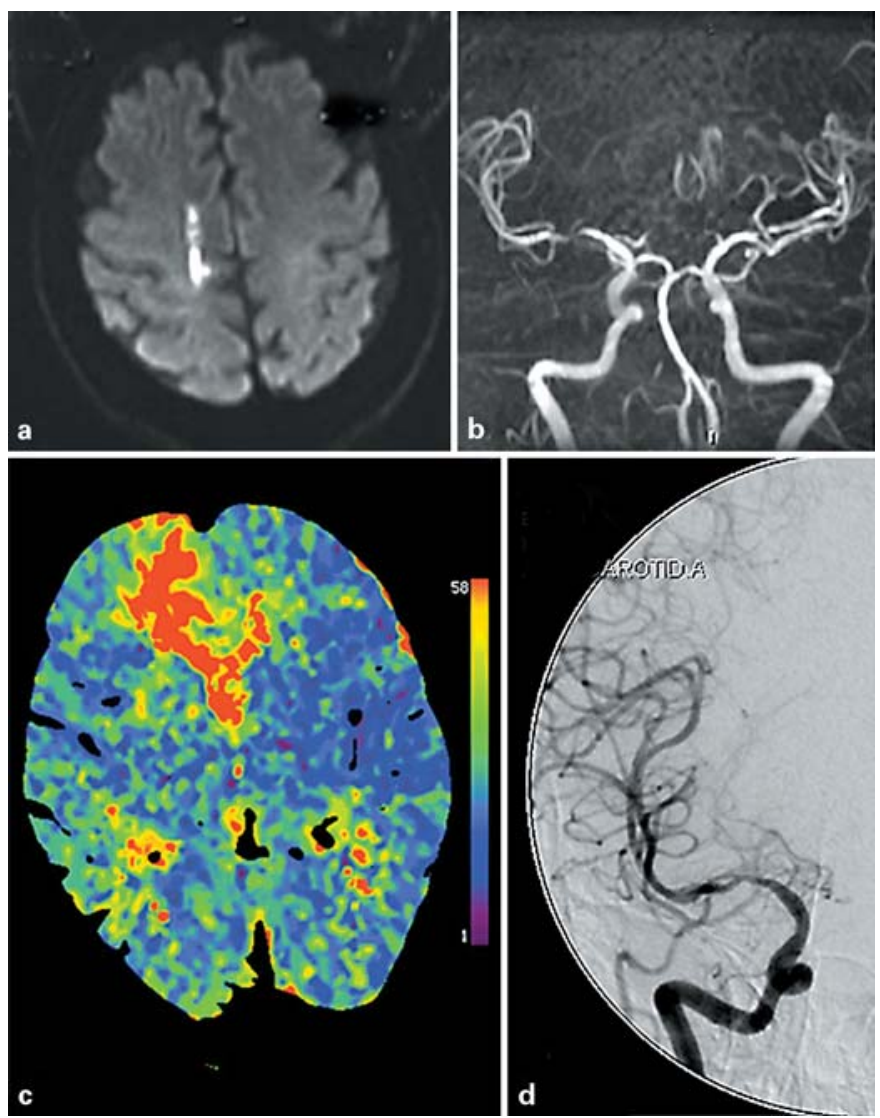

Fig. 1. a Diffusion-weighted MRI revealed high signal intensity lesions in the right watershed territory between the ACA and MCA. b Magnetic resonance angiogram showed severe focal stenosis of the right MCA and absence of bilateral ACA. c Perfusion CT showed delayed mean transit time in the right anterior frontal lobe, the right anterior and posterior border zone, and the right basal ganglia. d Digital substraction angiography showed severe focal stenosis of the M1 segment of the right MCA and absence of the ACA. The right border zone (between the ACA and MCA) shifted internally, suggestive of the right ACA territory being compensated partially by the leptomeningeal collateral vessels from the right MCA.

cerebral artery (MCA) (fig. 1a). Magnetic resonance angiogram showed severe focal stenosis of the right MCA and absence of bilateral ACA (fig. 1b). Perfusion CT showed delayed time to peak and mean transit time, increased relative cerebral blood flow, and slightly decreased relative cerebral blood flow in right anterior frontal lobe, the right anterior and posterior border zone and the right basal ganglia (fig. 1c). Digital substraction angiography showed severe focal stenosis of the M1 segment of the right MCA and absence of bilateral ACA. The right border zone (between ACA and MCA) was shifted internally, suggestive of the right ACA territory being compensated partially by the leptomeningeal collateral vessels from the right MCA (fig. 1d). Extracranial segment of the right internal carotid artery was normal. The patient was diagnosed as a case of 
transient ischemic attack (TIA), secondary to right MCA stenosis. The patient was treated with $300 \mathrm{mg}$ of acetylsalicylic acid and $75 \mathrm{mg}$ of clopidogrel once daily and advised to avoid sudden change in posture. Her blood pressure was maintained at a higher level (systolic blood pressure: $150-170 \mathrm{~mm} \mathrm{Hg}$, and diastolic blood pressure: $90-100 \mathrm{~mm} \mathrm{Hg}$ ) to avoid hypoperfusion. The follow-up evaluation after 3 months revealed significant improvement in the frequency and intensity of limb-shaking attacks. We recommended angioplasty and stenting of the right MCA, but the patient refused it.

\section{Discussion}

Limb-shaking TIA has been reported as an unusual type of TIA, most commonly caused by transient cerebral ischemia due to severe stenosis or occlusion of an extracranial internal carotid artery [1-3]. Recently, Han et al. [4] reported that ACA stenosis can also induce limb-shaking TIA. A shaking limb episode has been described as 'a brief, involuntary, coarse, irregular wavering movement or tremble' involving arm-hand alone or arm-hand and leg together [2].

The precise mechanism by which focal ischemia causes limb shaking is unknown. The generally accepted view is that the limbshaking TIA is caused by transient cerebral ischemia resulting from a low perfusion hemodynamic state due to severe extracranial and/ or intracranial carotid occlusive disease affecting primarily the border zone between the ACA and the MCA territory [5, 6]. Orthostatic position change, long standing position, neck extension, or hypotension induced by antihypertensive medication have been reported to trigger these involuntary movements, which suggests low perfusion hemodynamic mechanism $[1,5]$. Brain perfusion can be analyzed by several diagnostic methods, such as CT, MRI, SPECT and PET. Ultrasound may also be useful in assessing brain perfusion on bedside in critically ill patients by using bolus perfusion harmonic imaging [7]. We used perfusion CT, which demonstrated the hypoperfusion affecting the right border zone between the ACA and MCA. In this case, the 'criminal lesion' was the severe focal stenosis of the M1 segment of the right MCA and absence of bilateral ACA, which induced hypoperfusion in the border zone between the ACA and MCA. Previous studies also suggested that a poor leptomeningeal collateral circulation might be a precondition for limb-shaking TIA $[8,9]$.

To our knowledge this is the first case report in which limbshaking TIAs were caused by MCA stenosis. Our experience with this patient indicates that the combination of cerebral blood flow studies and cerebral angiography may facilitate identification of patients with limb-shaking TIA caused by intracranial artery stenosis.

\section{References}

1 Fisch BJ, Tatemichi TK, Prohovnik I, et al: Transient ischemic attacks resembling simple partial motor seizures. Neurology 1988;38(suppl 1):264.

2 Baquis GD, Pessin MS, Scott RM: Limb shaking - a carotid TIA. Stroke 1985; 16:444-448.

3 Klempen NL, Janardhan V, Schwartz RB, Stieg PE: Neurosurgery 2002; 51:483-487.

4 Han SW, Kim SH, Kim JK, Park CH, Yun MJ, Heo JH. Hemodynamic changes in limb shaking TIA associated with anterior cerebral artery stenosis. Neurology 2004:63:1519-1521.

5 Tatemichi TK, Young WL, Prohovnik I, Gitelman DR, Correll JW, Mohr JP: Perfusion insufficiency in limb-shaking transient ischemic attack. Stroke 1990;21:341-347.

6 Salah Uddin AB: Limb shaking transient ischemic attack - an unusual presentation of carotid occlusive disease. A case report and review of the literature. Parkinsonism Relat Disord 2004;10:451-453.

7 Seidel G, Wiethe KM, Berdien G, Hollstein D, Toth D, Phys D, Aach T, Ing D: Ultrasound perfusion imaging in acute middle cerebral artery infarction predicts outcome. Stroke 2004;35:1107-1111.

8 Zaidat OO, Werz MA, Landis DMD, Selman W: Orthostatic limb shaking from carotid hypoperfusion. Neurology 1999;53:650-651.

9 Khan A, Beletsky V, Kelley R, Ehsan T: Orthostatic-mediated hypoperfusion in limb-shaking transient ischemic attack. J Neuroimaging 1999;9: 43-44.

Feng Gao, MD

Department of Neurology and Neuroradiology

Beijing Tiantan Hospital, Capital University of Medical Sciences Beijing, 100050 (China)

E-Mail gaofengletter@hotmail.com 